EPJ Web of Conferences 109,05007 (2016)

DOI: 10.1051/epjconf/201610905007

(C) Owned by the authors, published by EDP Sciences, 2016

\title{
Photodisintegration of ${ }^{9} \mathrm{Be}$ and the importance of the unbound $1 / 2^{+}$state
}

\author{
Myagmarjav Odsuren ${ }^{1, a}$, Yuma Kikuchi ${ }^{2}$, Takayuki Myo ${ }^{3,4}$, Masayuki Aikawa ${ }^{5}$, and Kiyoshi Katō ${ }^{5}$ \\ ${ }^{1}$ School of Engineering and Applied Sciences, National University of Mongolia, Ulaanbaatar 210646, Mon- \\ golia \\ ${ }^{2}$ RIKEN Nishina Center, Wako, 351-0198, Japan \\ ${ }^{3}$ General Education, Faculty of Engineering, Osaka Institute of Technology, Osaka 535-8585, Japan \\ ${ }^{4}$ Research Center for Nuclear Physics (RCNP), Osaka University, Ibaraki 567-0047, Japan \\ ${ }^{5}$ Nuclear Reaction Data Centre, Faculty of Science, Hokkaido University, Sapporo 060-0810, Japan
}

\begin{abstract}
The photodisintegration cross section of the ${ }^{9} \mathrm{Be}\left(1 / 2^{+}\right)$state is investigated in a framework of the $\alpha+\alpha+n$ three-body model. The $1 / 2^{+}$state of ${ }^{9} \mathrm{Be}$ locates just above the three-body threshold, and the $s$-wave neutron property of this state is a long-standing problem. It has been a topic of quite interest in relation to the breakup mechanism of ${ }^{9} \mathrm{Be}$ into the ${ }^{8} \mathrm{Be}\left(0^{+}\right)+n$ and $(\alpha+\alpha+n)$ channels.
\end{abstract}

\section{Introduction}

The elements heavier than iron are mainly produced by the $r$-process in supernovae [1]. The ${ }^{8} \mathrm{Be}(n, \gamma){ }^{9} \mathrm{Be}$ is one of the significant reaction in the neutron rich $r$-process [2]. Buchmann et al. [3] discussed the calculation of this reaction rate. They reported that the stellar reaction rate of ${ }^{8} \mathrm{Be}(n, \gamma){ }^{9} \mathrm{Be}$ depends strongly on the properties of the $1 / 2^{+}$state of ${ }^{9} \mathrm{Be}$ just located above the $\alpha+\alpha+n$ three-body threshold energy. The first excited ${ }^{9} \mathrm{Be}\left(1 / 2^{+}\right)$state has been investigated in various types of experiment. The energy of the $1 / 2^{+}$for the unbound state was suggested based on electron scattering [4], proton scattering [5] and photodisintegration [6,7].

The measurement of the ${ }^{8} \mathrm{Be}(n, \gamma)^{9} \mathrm{Be}$ reaction cross section is not able since there does not exist a stable configuration with ${ }^{8} \mathrm{Be}$ (the lifetime of ${ }^{8} \mathrm{Be}$ is very short about $10^{-16} \mathrm{~s}$ ). Inversely, the ${ }^{8} \mathrm{Be}(n, \gamma){ }^{9} \mathrm{Be}$ reaction cross section can be deduced from the inverse ${ }^{9} \mathrm{Be}(\gamma, n){ }^{8} \mathrm{Be}$ reaction. The measured photodisintegration cross sections of the $1 / 2^{+}$state of ${ }^{9} \mathrm{Be}$ in Refs. [6, 8] are inconsistent with recent new experimental value [7]. Although many studies have been performed, the structure of the ${ }^{9} \mathrm{Be}\left(1 / 2^{+}\right)$state by using various theoretical models has not been settled yet.

The purpose of this work is to discuss the structure of the ${ }^{9} \mathrm{Be}\left(1 / 2^{+}\right)$state. We apply the complex scaling method (CSM) [9-12] to an $\alpha+\alpha+n$ three-body model to investigate the structure of the $1 / 2^{+}$state. The low-lying peak in the photodisintegration cross section of the ${ }^{9} \mathrm{Be}\left(1 / 2^{+}\right)$is calculated and compared with the recent measurement [7]. To explain the low-energy peak in the calculated photodisintegration cross section, each component (two- and three-body continuum states) in the three-body contributions to the total cross section is calculated. In order to see the effect of each

\footnotetext{
ae-mail: odsurenn@gmail.com
} 
component on the total cross section clearly, we calculate the decomposed $E 1$ transition strengths for the $1 / 2^{+}$state. In particular, we focus on the contributions of the ${ }^{9} \mathrm{Be}\left(1 / 2^{+}\right)$state.

It is particular interest to compare the structure of the ${ }^{9} \mathrm{Be}\left(1 / 2^{+}\right)$state with another higher excited state of ${ }^{9} \mathrm{Be}$. For this purpose, the structure study of the $1 / 2^{+}$and $5 / 2^{+}$is useful. For the $1 / 2^{+}$state, the eigenvalue distribution of the low-lying $1 / 2^{+}$state is calculated in CSM but a resonance energy is not obtained. We find a peak in photodisintegration cross section using the $\alpha+\alpha+n$ three-body model, which agrees well with the newly measured data [7]. On the other hand, the $5 / 2^{+}$state, a resonance energy and the contribution to the $E 1$ strength are calculated.

\section{Theoretical framework}

\subsection{Three-body model}

The Hamiltonian for the relative motion of the $\alpha+\alpha+n$ three-body system for ${ }^{9} \mathrm{Be}$ is given as

$$
\hat{H}=\sum_{i=1}^{3} t_{i}-T_{\text {c.m. }}+\sum_{i=1}^{2} V_{\alpha n}\left(\xi_{i}\right)+V_{\alpha \alpha}+V_{\mathrm{PF}}+V_{3},
$$

where $t_{i}$ and $T_{\text {c.m. }}$ are kinetic operators for each particle and the center-of-mass of the system, respectively. The interactions between the neutron and the $i$-th $\alpha$ particle are given as $V_{\alpha n}\left(\boldsymbol{\xi}_{i}\right)$, where $\boldsymbol{\xi}_{i}$ is the relative coordinate between them. We here employ the KKNN potential [13] for $V_{\alpha n}$ subsystem. The $V_{\alpha \alpha}$ is the potential of a subsystem for the $\alpha+\alpha$ interaction and we apply a folding potential [14] of the effective nuclear and Coulomb forces. The three-body potential $V_{3}$ is explicitly given as

$$
V_{3}=v_{3} b \exp \left(-\mu \rho^{2}\right)
$$

where $\rho$ is the hyper-radius of the $\alpha+\alpha+n$ three-body system. In the present calculation, we use the pseudo potential $V_{\mathrm{PF}}$ to take account of the Pauli principle between clusters.

We investigate the properties of the unbound states of ${ }^{9} \mathrm{Be}$ by using the CSM. The CSM has been applied to investigate many-body resonance states and study of its structures. The complex-scaled Schrödinger equation is expressed using the complex-scaled Hamiltonian $\hat{H}^{\theta}$ as

$$
\hat{H}^{\theta} \Psi_{J}^{v}(\theta)=E \Psi_{J}^{v}(\theta)
$$

where $J$ is the total spin of the $\alpha+\alpha+n$ and $v$ is the state index. The Jacobi coordinates $\vec{r}_{i}$ for the relative motion of the $\alpha+\alpha+n$ three-body system are transformed as $\vec{r}_{i} \rightarrow \vec{r}_{i} e^{i \theta}(i=1,2)$ with a real parameter $\theta$. The $U(\theta)$ operates on a function $\Psi_{J}^{v}$, that is,

$$
\Psi_{J}^{v}(\theta)=U(\theta) \Psi_{J}^{v}(r)=e^{\frac{3}{2} i \theta} \Psi_{J}^{v}\left(r e^{i \theta}\right)
$$

The complex scaled Hamiltonian of inter cluster motion is given by

$$
\hat{H}(\theta)=U(\theta) \hat{H} U^{-1}(\theta) .
$$

\subsection{Photodisintegration cross section}

Photodisintegration cross section $\sigma_{\gamma n}$

$$
{ }^{9} \mathrm{Be}\left(3 / 2^{-}\right)+\gamma \rightarrow n+\alpha+\alpha,
$$


Table 1. The calculated binding energies, charge- and matter radii with and without three-body potential are given. ${ }^{a}$ Reference [15]; ${ }^{b}$ Reference [16]; ${ }^{c}$ Reference [17].

\begin{tabular}{llllll}
\hline & $E_{\text {g.s. }}[\mathrm{MeV}]$ & $R_{c h}[\mathrm{fm}]$ & $R_{m}[\mathrm{fm}]$ & $v_{3 b}[\mathrm{MeV}]$ & $\mu\left[\mathrm{fm}^{-2}\right]$ \\
\hline with $V_{3}$ & 1.57 & 2.53 & 2.42 & 1.10 & 0.02 \\
without $V_{3}$ & 2.14 & 2.50 & 2.39 & - & - \\
Exp. & $1.5736^{a}$ & $2.519 \pm 0.012^{b}$ & $2.38 \pm 0.01^{c}$ & & \\
\hline
\end{tabular}

calculated in terms of the multi-pole response which can be expressed as the following form

$$
\sigma_{E \lambda}^{\gamma}\left(E_{\gamma}\right)=\frac{(2 \pi)^{3}(\lambda+1)}{\lambda[(2 \lambda+1) ! !]^{2}}\left(\frac{E_{\gamma}}{\hbar c}\right)^{2 \lambda-1} \frac{d B\left(E \lambda, E_{\gamma}\right)}{d E_{\gamma}}
$$

In this work, we consider the $E 1$ transitions from the $3 / 2^{-}$ground state to the unbound states in ${ }^{9} \mathrm{Be}$ using the complex-scaled Green's function, which contribute to the ${ }^{9} \mathrm{Be}(\gamma, n)^{8} \mathrm{Be}$ cross section as

$$
\sigma_{E 1}^{\gamma}\left(E_{\gamma}\right)=\frac{16 \pi^{3}}{9 \hbar c} E_{\gamma} \frac{d B\left(E 1, E_{\gamma}\right)}{d E_{\gamma}}
$$

\section{Results}

The calculated charge radii, matter radii and binding energy of ${ }^{9} \mathrm{Be}$ are shown in Table 1 . These quantities of the ${ }^{9} \mathrm{Be}\left(3 / 2^{-}\right)$ground state is calculated from the $\alpha+\alpha+n$ three-body threshold with $\left(v_{3 b}=1.10 \mathrm{MeV}, \mu=0.02 \mathrm{fm}^{-2}\right)$ and without $\left(v_{3 b}=0\right)$ three-body potential. The experimental data are taken from Refs. [15-17]. We can see a good agreement between our calculated results with three-body potential and experimental data. This indicates that these quantities of the ${ }^{9} \mathrm{Be}$ ground state are well established by $v_{3 b}=1.10 \mathrm{MeV}, \mu=0.02 \mathrm{fm}^{-2}$.

The recent measurement of the ${ }^{9} \mathrm{Be}(\gamma, n)$ reaction using nearly mono-energetic photon beams reported that the photodisintegration cross section rises sharply to the peak just above the ${ }^{8} \mathrm{Be}\left(0^{+}\right)+n$ threshold [7]. To explain this feature of the photodisintegration, we employ a three-body $\alpha+\alpha+n$ model by adding the three-body potential. To discuss the observed sharp peak just above the ${ }^{8} \mathrm{Be}\left(0^{+}\right)+n$ threshold in the photodisintegration cross section, we change the strength $v_{3 b}$ for the $1 / 2^{+}$state. The range $\mu$ is fixed as the same as used in the ground state for all calculations. With a negative (attractive) strength of the three-body potential $v_{3 b}=-1.02 \mathrm{MeV}$, we can reproduce newly measured cross section as shown in Fig. 1. We find that the calculated cross sections show the dependence on the strength of the three-body potential as shown in Fig. 1. This result is interesting and suggests the existence of the three-body unbound state of ${ }^{9} \mathrm{Be}\left(1 / 2^{+}\right)$, such as a resonance or virtual state. In relation to the cross section, we discuss the character of the $1 / 2^{+}$state.

We investigate the origin of the low-lying peak above the ${ }^{8} \mathrm{Be}\left(0^{+}\right)+n$ threshold in detail. For this purpose, we show the distribution of the energy eigenvalues of the $1 / 2^{+}$states by using the CSM. The Eq. (1) reproduces all the threshold energies of $\alpha+\alpha+n,{ }^{8} \mathrm{Be}+n$ and ${ }^{5} \mathrm{He}+\alpha$ in ${ }^{9} \mathrm{Be}$. Using Eq. (1) with three-body potential $v_{3 b}=-1.02 \mathrm{MeV}, \mu=0.02 \mathrm{fm}^{-2}$ parameters, we do not obtain the $1 / 2^{+}$ resonance of ${ }^{9} \mathrm{Be}$ as shown in Fig. 2. But it reproduces the observed peak as shown in Fig. 1.

The advantage of the CSM is to decompose the unbound states into resonant and nonresonant continuum states by rotating the branch cut on the complex energy plane with a parameter $\theta$. As a result, we can separate a physical quantity into two parts associated with resonant and nonresonant continuum states. Using the CSM, we decompose the $E 1$ transition strength calculated with $v_{3 b}=$ $-1.02 \mathrm{MeV}$ into ${ }^{8} \mathrm{Be}\left(0^{+}\right)+n$ and $\alpha+\alpha+n$ components for the $1 / 2^{+}$state as shown in Fig. 3(a), to 


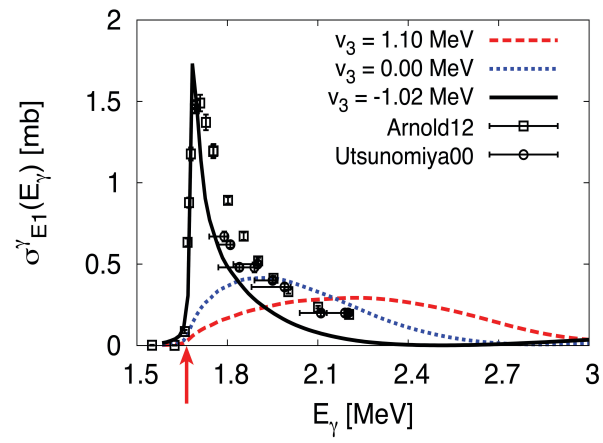

Figure 1. Calculated photodisintegration cross sections in comparison with the experimental data. The experimental data are taken from Refs. [6] and [7]. The arrow indicates the threshold energy of the ${ }^{8} \mathrm{Be}\left(0^{+}\right)+n$ channel. The red (dashed) and blue (dotted) lines are results with and without the three-body potential, where $v_{3 b}=1.10 \mathrm{MeV}$ and $\mu=0.02 \mathrm{fm}^{-2}$. The black (solid) line represents the cross section calculated by using an attractive three-body potential with $v_{3 b}=-1.02 \mathrm{MeV}$.

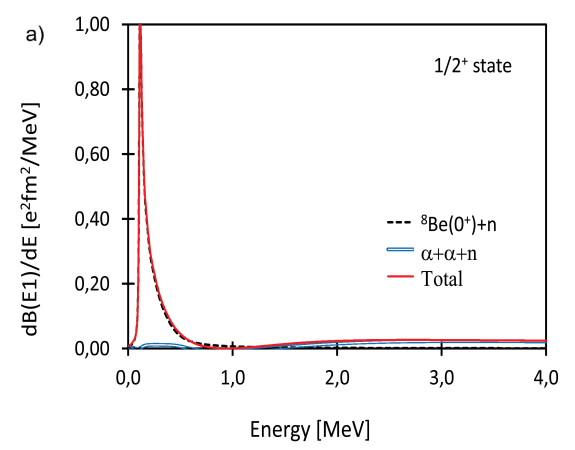

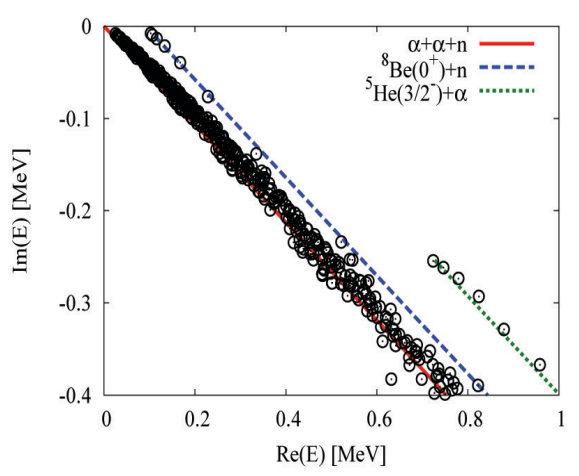

Figure 2. Distribution of energy eigenvalues of the $J^{\pi}=1 / 2^{+}$states measured from the $\alpha+\alpha+n$ threshold with scaling angle $\theta=15$ degrees. The three-body potential with $v_{3 b}=-1.02 \mathrm{MeV}$ and $\mu=0.02 \mathrm{fm}^{-2}$ is applied. The red (solid), blue (dashed), and green (dotted) lines represent the branch cuts for $\alpha+\alpha+n,{ }^{8} \operatorname{Be}\left(0^{+}\right)+n$, and ${ }^{5} \mathrm{He}\left(3 / 2^{-}\right)+\alpha$ continua, respectively

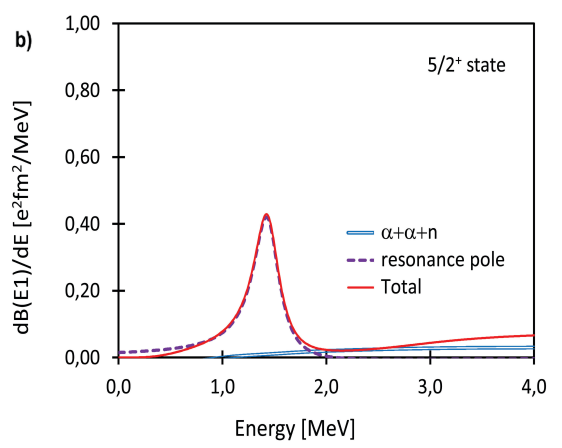

Figure 3. Decomposed $E 1$ transition strengths for the (a) $J^{\pi}=1 / 2^{+}$and (b) $J^{\pi}=5 / 2^{+}$states. The red (solid) and blue (open) lines are of the total $E 1$ and $\alpha+\alpha+n$ contributions. The dashed violet lines in (a) and (b) denote the results of ${ }^{8} \mathrm{Be}\left(0^{+}\right)+n$ continuum and resonance contributions, respectively.

see the dominant contribution in the cross section [18]. The ${ }^{5} \mathrm{He}\left(3 / 2^{-}\right)+\alpha$ contribution is found to be negligible in the low-lying region, and we do not show it in Fig. 3(a). From Fig. 3(a), we see that the ${ }^{8} \mathrm{Be}\left(0^{+}\right)+n$ component is almost identical to the total cross section. This fact indicates that the ${ }^{8} \mathrm{Be}\left(0^{+}\right)+n$ decay is dominant in the photodisintegration. This decay mode should be related to the structure of the $1 / 2^{+}$state of ${ }^{9} \mathrm{Be}$.

Including calculations of the ${ }^{9} \mathrm{Be}\left(5 / 2^{+}\right)$state, we try to understand structure of the ${ }^{9} \mathrm{Be}\left(1 / 2^{+}\right)$state. To see the structure of the ${ }^{9} \mathrm{Be}\left(5 / 2^{+}\right)$state, the decomposed $E 1$ transition strength is quite important 


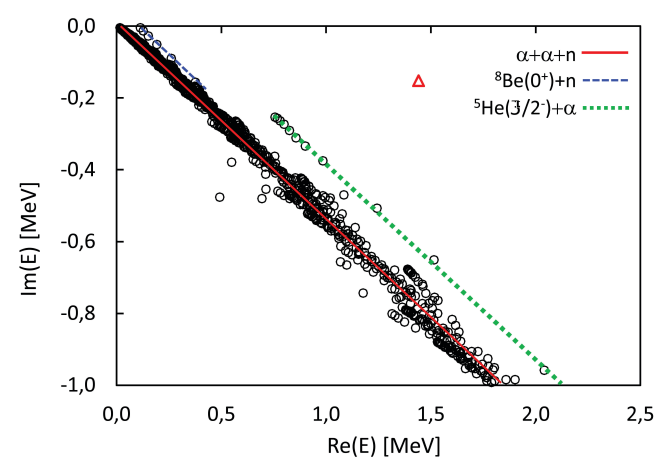

Figure 4. Distribution of energy eigenvalues of $J=5 / 2^{+}$states measured from the $\alpha+\alpha+n$ threshold with scaling angle $\theta=15$ degrees. The three-body potential with $v_{3 b}=-0.3 \mathrm{MeV}$ and $\mu=0.02 \mathrm{fm}^{-2}$ is applied. The red (solid), blue (dashed), and green (dotted) lines are same as that in Fig. 2. The red triangle is obtained resonant pole.

to see the dominant contribution in the cross section. The decomposed $E 1$ transition strengths of the ${ }^{9} \mathrm{Be}\left(5 / 2^{+}\right)$are shown in Fig. 3(b). This result indicates that the obtained resonance state gives a dominant contribution to the total $E 1$ transition strength. The ${ }^{5} \mathrm{He}\left(3 / 2^{-}\right)+\alpha$ contribution is negligible, similarly to the $1 / 2^{+}$case, and it is not shown in Fig. 3(b).

The complex energy of resonant state is obtained as $E^{\theta}=E_{r}-i \Gamma / 2$, when $\tan ^{-1}\left(\Gamma / 2 E_{r}\right)<2 \theta$. Using Eq. (1) and with $v_{3 b}=-0.3 \mathrm{MeV}$ strength, we solve the complex scaled eigenvalue problem for the ${ }^{9} \mathrm{Be}\left(5 / 2^{+}\right)$and is presented in Fig 4. One resonant pole of the ${ }^{9} \mathrm{Be}\left(5 / 2^{+}\right)$state is obtained as $\left(E_{r}, \Gamma\right)=(1.47,0.32) \mathrm{MeV}$, which agree with the experimental data [15] $(1.475,0.282) \mathrm{MeV}$. Using this result, we calculate the $E 1$ transition strength, which is shown in Fig. 3 (b).

\section{Discussions and Summary}

The character of the ${ }^{9} \mathrm{Be}\left(1 / 2^{+}\right)$state is investigated by using the photodisintegration reaction with the $\alpha+\alpha+n$ three-body model and the CSM. The calculated photodisintegration cross sections into the $1 / 2^{+}$states are shown to have dependence on the strength of the three-body potential for the $1 / 2^{+}$ state. The experimental cross section shows the sharp peak just above the ${ }^{8} \mathrm{Be}\left(0^{+}\right)+n$ threshold, which is nicely reproduced with the attractive three-body potential. We do not obtain any resonance poles for the $1 / 2^{+}$states in explaining the peak in the cross section. From the decomposition of the calculated cross section, it is shown that the ${ }^{8} \mathrm{Be}\left(0^{+}\right)+n$ continuum states dominates the cross section to the $1 / 2^{+}$ states. We also calculate the ${ }^{9} \mathrm{Be}\left(5 / 2^{+}\right)$state and a resonance energy is obtained. The decomposed $E 1$ transition strengths of the ${ }^{9} \mathrm{Be}\left(5 / 2^{+}\right)$state are indicated that the obtained resonance state is given dominant contribution to the total $E 1$ transition strength. These results indicate the possibility of the virtual-state nature of the first excited $1 / 2^{+}$state of ${ }^{9} \mathrm{Be}$.

\section{References}

[1] E.M, Burbidge, G.R. Burbidge, W.L. Fowler, F. Hoyle, Reviews of Modern Physics, 29, 547, (1957). 


\section{EPJ Web of Conferences}

[2] T. Sasaqui, K.T. Kajino, G.J. Mathews, K. Otsuki, T. Nakamura, Astrophys. J. 634, 1173 (2005).

[3] L. Buchmann, E. Gete, J. C. Chow, J. D. King, D. F. Measday, Phys. Rev. C 63, 034303, (2001).

[4] J. P. Glickman et. al., Phys. Rev. C 43, 1740 (1991).

[5] S. Dixit et al., Phys. Rev. C 43, 1758, (1991).

[6] H. Utsunomiya, Y. Yonezawa, H. Akimune, T. Yamagata, M. Ohta, M. Fujishiro, H. Toyokawa, H. Ohgaki, Phys. Rev. C 63, 018801 (2000).

[7] C.W. Arnold, T.B. Clegg, C. Iliadis, H.J. Karwowski, G.C. Rich, J.R. Tompkins, C.R. Howell, Phys. Rev. C 85, 044605, (2012).

[8] K. Sumiyoshi, H. Utsunomiya, S. Goko, and T. Kajino, Nucl. Phys. A 709, 467 (2002).

[9] K. Arai, P. Descouvemont, D. Baye, and W. N. Catford, Phys. Rev. C 68, 014310 (2003).

[10] E. Garrido, D. Fedorov, and A. Jensen, Phys. Lett. B 684, 132 (2010).

[11] Y.K. Ho, Phys. Rep. 99, 1 (1983).

[12] T. Myo, Y. Kikuchi, H. Masui, K.Katō, Prog. Part. Nucl. Phys. 79, 1 (2014).

[13] H. Kanada, T. Kaneko, S. Nagata, and M. Nomoto, Prog. Theor. Phys. 61, 1327 (1979).

[14] E. Schmid and K. Wildermuth, Nuclear Physics 26, 463 (1961).

[15] D. Tilley, J. Kelley, J. Godwin, D. Millener, J. Purcell, C. Sheu, and H. Weller, Nucl. Phys. A 745, 155 (2004).

[16] W. Noertershaeuser, et al., Phys. Rev. Lett. 102, 062503 (2009).

[17] I. Tanihata, T. Kobayashi, O. Yamakawa, S. Shimoura, K. Ekuni, K. Sugimoto, N. Takahashi, T. Shimoda, H. Sato, Phys. Lett. B 206, 592 (1988).

[18] T. Myo, K. Katō, S. Aoyama, and K. Ikeda, Phys. Rev. C 63, 054313 (2001). 